AperTO - Archivio Istituzionale Open Access dell'Università di Torino

\title{
New perspectives for mitotane treatment of adrenocortical carcinoma
}

\section{This is the author's manuscript}

Original Citation:

Availability:

This version is available http://hdl.handle.net/2318/1743740

since 2022-10-11T12:50:29Z

Published version:

DOI:10.1016/j.beem.2020.101415

Terms of use:

Open Access

Anyone can freely access the full text of works made available as "Open Access". Works made available under a Creative Commons license can be used according to the terms and conditions of said license. Use of all other works requires consent of the right holder (author or publisher) if not exempted from copyright protection by the applicable law. 


\section{NEW PERSPECTIVES FOR MITOTANE TREATMENT OF ADRENOCORTICAL CARCINOMA.}

S. Puglisi, A. Calabrese, V. Basile, A. Pia, G. Reimondo, P. Perotti, M. Terzolo. Internal Medicine, Department of Clinical and Biological Sciences, S. Luigi Gonzaga Hospital, University of Turin, Orbassano, Italy

Corresponding author:

Prof. Massimo Terzolo

Internal Medicine, Dept. of Clinical and Biological Sciences, University of Turin

S. Luigi Gonzaga Hospital, Regione Gonzole 10, Orbassano (TO) 10043, Italy (+39) 0116705457

email: massimo.terzolo@unito.it 


\begin{abstract}
Adrenocortical carcinoma (ACC) is an aggressive cancer characterized by poor survival. Apart from radical surgery, there is a limited range of therapeutic options and mitotane remains the cornerstone of medical treatment of ACC in either adjuvant or palliative settings. The aim of adjuvant mitotane therapy is to reduce the risk of ACC recurrence following surgical removal of the tumor. Use of mitotane in an adjuvant setting is off-label, but the recent guidelines endorsed by the European Society of Endocrinology (ESE) and the European Network for the Study of Adrenal Tumors (ENSAT) recommend it in ACC patients at high risk of recurrence. The palliative use of mitotane for treatment of advanced ACC aims at controlling tumor progression and, when present, hormone secretion. In this clinical setting, mitotane is used in association with chemotherapy to treat the more aggressive forms, while mitotane monotherapy is reserved for less progressive ACC. Many years after its introduction in clinical practice, there are still uncertainties surrounding the use of this old drug and the derived benefits. Moreover, physicians who use mitotane should recognize and manage the systemic effects of the drug that need a complex supporting therapy.
\end{abstract}

\title{
Keywords
}

Adjuvant therapy, adrenal cancer, cortisol, chemotherapy, Cushing syndrome, mitotane. 


\section{Introduction}

Adrenocortical carcinoma (ACC) is extremely rare, with a reported incidence of about two cases per million per year. ACC is more frequently detected in women (55-60\%) and in the fourth and fifth decades; however, any age including childhood can be affected $(1,2)$.

ACC is an aggressive cancer with an overall 5-year survival rate between $16 \%$ and $47 \%(3,4)$. Prognosis is mainly influenced by tumor stage, being the 5 -year survival $81,61,50$, and $13 \%$, respectively, from stage I to stage IV, and the possibility to remove completely the tumor, which also depends on ACC stage at diagnosis (5). However, ACC is a heterogeneous disease and survival may show considerable variability at any stage depending on molecular, pathological and clinical factors that have only been partially elucidated (6).

One of the factors influencing the clinical phenotype of ACC patients is the functional activity of the tumor, which may result in different endocrine syndromes $(3,4,6)$. Manifestations of adrenal steroid hormone excess represent a common presentation of ACC that may be found in up to $60 \%$ of cases $(3,4,7)$. Patients with nonfunctioning ACC present with back or abdominal pain, nausea, vomiting, or less frequently fever and weigh loss. In an increasing number of patients, ACC is discovered serendipitously, due to the widespread application of high-resolution crosssectional scans (8).

A limited range of therapeutic options is available for ACC. The rarity and aggressiveness of the tumor have hindered progress in treatment that remains mainly based on surgery. Surgery is usually the first and most effective therapeutic strategy, while survival is poor when surgical removal of ACC is unfeasible (9-11). Complete surgery may be curative; however, the odds that ACC may recur after surgery are high $(11,12)$. Tumor recurrence can be explained with technical failure of surgery leading to tumor rupture or spillage, but is also frequently observed after microscopically radical (R0) operations done by skilled surgeons $(11,12)$. Therefore, a sound treatment strategy should include in principle an adjuvant treatment to avoid tumor recurrence. Administration of mitotane has been the most followed adjuvant approach in clinics, albeit mitotane is formally approved by regulatory agencies for treatment of advanced ACC $(2,3)$.

In patients with inoperable or metastatic disease, mitotane is the mainstay of treatment and can be used as a single agent or in combination with classic cytostatic drugs. A 
key concept of mitotane treatment in patients with advanced/metastatic ACC is that disease responses are mainly seen in patients whose plasma mitotane concentrations are between 14 and $20 \mathrm{mg} / \mathrm{L}(2-4,6)$.

Owing to the latency to attain the therapeutic range, mitotane monotherapy may best suited for patients with a low tumor burden and less rapid disease. For ACC showing an aggressive disease course, or with many metastatic sites, cytotoxic chemotherapy in association with mitotane may be more reasonable, particularly when ACC is progressing on mitotane therapy $(2-4,6)$. The polychemotherapy regimen EDP-M, including etoposide, doxorubicin, cisplatin and mitotane, represents the current standard of chemotherapy for advanced ACC, being the only treatment strategy supported by a randomized controlled trial (13).

The first report of the capability of mitotane to destroy the zona fasciculata and zona reticularis in the adrenal glands was published in 1949, showing that mitotane treatment in dogs induced a marked reduction of glucocorticoids and 17hydroxycorticosteroids either in basal conditions or after ACTH stimulation $(14,15)$. Following this observation, it was found that mitotane is able to inhibit gene expression of a variety of enzymes of the steroidogenetic pathway (15). This leads to a remarkable inhibition of adrenal steroid production; thus, mitotane may ameliorate signs and symptoms of cortisol excess, and this adrenolytic drug has been used as a medical treatment of all causes of Cushing's syndrome $(16,17)$.

More than 50 years after the introduction in clinical practice of mitotane, we still do not know precisely its mechanism of action, what is the optimal use of this old drug and what we may expect in terms of efficacy. Mitotane is currently used both in postoperative adjuvant and palliative (advanced) settings; however, no data from randomized prospective trials on mitotane as monotherapy are available to guide management. 


\section{Mechanism of action of mitotane}

Mitotane, [1,1-dichlorodiphenildichloroethane (o,p'-DDD)], a parent compound of the insecticide dichlorodiphenyltrichloroethane (DDT), is the only drug approved by the FDA and EMEA for ACC treatment $(2-4,6)$. Mitotane has a profound effect on steroidogenesis $(16,17)$ that may be explained with the inhibition of gene transcription of many steroidogenic enzymes (18-20). Steroidogenic acute regulatory protein (StAR) and CYP11A1, which are involved in the rate-limiting step of steroidogenesis, are most sensitive to mitotane (19). The intracellular targets of mitotane remains to be identified; however, mitotane has been found to produce mitochondrial damage that activates an apoptotic process involving caspase 3 and caspase 7 activities (20). Studies showed that mitotane is able to interfere with the mitochondrial respiratory chain function complex IV (cytochrome coxidase) and to induce morphologic fragmentation of the mitochondrial membranes that are required for respiratory chain activity and presumably steroidogenesis (21).

Moreover, mitotane was found to be a strong inducer of CYP3A4 activity leading to glucocorticoid inactivation and a consequent sharp rise in 6 $\beta$-hydroxycortisol urinary excretion. It was calculated that mitotane is able to inactivate $50 \%$ of administered hydrocortisone, thus explaining why patients on mitotane do need higher doses of steroids for an effective replacement (22).

Study demonstrated that mitotane is an inhibitor of sterol-O-acyl-transferase 1 (SOAT1) leading to accumulation of free cholesterol that becomes toxic for the cell. The fact that SOAT1 is predominantly expressed by the adrenals explains why mitotane, by inhibiting SOAT1, down-regulates steroidogenesis and exerts a cytotoxic effect due to lipid-induced endoplasmic reticulum stress. In a small number of ACC tissues, SOAT1 expression correlated with the response to mitotane treatment, i.e. low SOAT1 expression was associated with poor response (23).

\section{Practice Point}

- Mitotane interferes with mitochondrial function reducing cell viability and function (hormone secretion).

\section{Research Agenda}

- The molecular targets of mitotane need to be elucidated to get a potential predictor of response that could be searched for in tumor cells. 


\section{Mitotane in the adjuvant setting}

The best outcome for ACC patients is dependent on the possibility of a radical surgery. Therefore, surgery must be performed by an experienced surgical team, in high volume centers $(12,24)$, with the goal of achieving R0 resection (microscopically free margins). The risk for recurrence is lower for patients who undergo surgery by expert surgeons (24), although the risk is never abolished. More than $50 \%$ of the tumors that have been completely extirpated are doomed to relapse (11,24-27), and most patients with ACC recurrence experience further tumor progression and eventually die. Surgical failure is not the only reason for recurrence, given that even stage I-II ACC recurs frequently. Several potential predictive factors of recurrence in radically resected ACC have been identified (28-31), but the issue of defining prognostic factors is complicated by the great variability of clinical presentation and biological heterogeneity of ACC.

The significant propensity of ACC to recur provides a strong rationale for adjuvant therapy following ACC removal. The pioneering work by Schteingart (32) prompted many researches to consider the use of post-operative mitotane as adjuvant therapy; however, evidence is still conflicting for a variety of reasons.

First, most studies had limited statistical power (26, 33-35). Second, many studies did not include a concomitant matched control group of untreated patients (26, 32, 34-37), whereas in some series a number of patients underwent multiple adjuvant treatments (38). In addition, the definition of recurrence-free survival (RFS) has not been uniform, and the duration of response has been sometimes unclear. Finally, all studies but one (32) were retrospective and employed different formulations of mitotane at doses ranging from 3 to $20 \mathrm{~g}$ daily, which were given for different periods (38).

Mitotane has a narrow therapeutic index and can cause significant toxicity and this does not make mitotane an ideal drug to treat patients free of disease. The potential of severe toxicity coupled with a controversial literature $(26,33,37-43)$ made adjunctive treatment with mitotane less appealing until the last 10 years.

Interest in adjuvant mitotane was resumed in 2007, when we published a retrospective analysis involving a large cohort of 177 ACC patients, followed for up to 10 years at different institutions in Italy and Germany. We found that patients treated with adjuvant mitotane had a significantly survival advantage compared with patients left untreated following surgery (44). The study challenged the concept that adjuvant mitotane therapy is unfeasible and ineffective; a favorable effect was achieved with low doses of 
mitotane (1-5 g per day), which were associated with acceptable toxicity (44). Conversely, severe and disabling toxicity was observed in previous studies employing high doses of mitotane (38). The retrospective nature of our study, however, does not allow concluding definitively that adjuvant mitotane treatment is beneficial. Arguments against adjuvant mitotane are mainly based on the methodological flaws of a retrospective analysis, toxicity and complexity of mitotane treatment, and lack of factors predicting response to treatment (45).

Novel evidence on the value of adjuvant mitotane has been published in the last years (46-48). A surgical study from the M.D. Anderson Cancer Center showed that the lack of adjuvant mitotane treatment was a factor predicting a higher risk of recurrence, despite the claim that a state-of-the-art surgical approach may avoid use of adjuvant mitotane (46). A study from Germany found that survival was improved in patients with stage II ACC managed at expert centers, in which adjuvant mitotane was used more frequently than in the community (47). A retrospective analysis from the University of Michigan reported on a large cohort of patients from a single center that mitotane treatment was associated with a significantly improved RFS. Mitotane was of benefit despite the fact that patients treated adjuvantly had a worse risk profile than the control group. A positive interaction between mitotane therapy and local radiotherapy was found, thus introducing the concept of a combined treatment (48).

The ESE-ENSAT guidelines suggest considering radiation in addition to mitotane therapy as an adjuvant measure on an individualized basis therapy in patients with R1 or Rx resection, or in stage III ACC (49).

Our group has updated the follow-up of the patient cohorts included in the 2007 study with almost 10 years of additional observation, confirming that adjuvant mitotane treatment was associated with a significant benefit in terms of RFS regardless of the hormone secretory status (50). Median RFS was 42 months in the adjuvant group compared with 17 months in control group $1(p<0.001)$ and 26 months in control group $2(p<0.005)$. Mitotane-treated patients had a significant benefit in overall survival (OS) in comparison with the control group 1, while the difference in OS just failed to reach statistical significance with the control group 2 (50). In this study, landmark analysis was employed to circumvent the immortal-time bias that is a common confounder of this type of studies. Despite its retrospective nature, this study remains the most informative one on the topic and represents a reference for decision making on adjuvant therapy. The advantage of our study compared to others is that treatment 
assignment was center-specific (i.e. all patients of a given center were treated or not treated) and not based on patient characteristics. In most studies, presence of unfavorable prognostic characteristics was a factor supporting the decision to give adjuvant mitotane and this have introduced confounding by indication since mitotanetreated patients had higher risk of recurrence at baseline than untreated patients.

Literature is conflicting and there is also recent evidence of a relative ineffectiveness of adjuvant mitotane. A retrospective analysis of the outcomes of 207 ACC patients, who underwent resection at 13 surgical centers in the United States, reported that adjuvant mitotane use was associated with decreased RFS (51). The difference with our results may be readily explained by the selection to mitotane treatment of patients at unfavorable prognosis in that study. The patients who were treated with mitotane had a higher frequency of stage IV metastatic tumors and, indeed, chemotherapy was frequently associated to mitotane therapy. Also, the frequency of cortisol excess, another negative prognostic factor, was higher in the mitotane group (51).

Efficacy of adjuvant mitotane treatment has been the subject of two recent metaanalyses that were able to retrieve only 6 and 5 studies, respectively, fulfilling the quality criteria of sufficient patient number, presence of a concomitant control group of untreated patients and reporting of baseline patient characteristics. Due to the variable inclusion criteria and different study procedures, a remarkable heterogeneity between studies was apparent in both meta-analyses that were not able to identify predictors of response to adjuvant mitotane $(49,52)$. Both meta-analyses, however, concluded that adjuvant mitotane was associated with a significant prolongation of OS, and in one study also RFS was significantly prolonged (52).

The ESE - ENSAT guidelines on the management of ACC suggest adjuvant mitotane treatment for patients at high risk of recurrence following complete tumor extirpation, although recognizing the low level of evidence of this recommendation. Adjuvant mitotane remains an option to be discussed on individual basis for patients at low risk of recurrence (49).

We have recently reviewed our experience with adjuvant mitotane treatment in nonmetastatic ACC patients following complete tumor resection (53). We did a retrospective analysis of 152 patients who have not been included in previous studies. Of those, 100 patients underwent adjuvant mitotane therapy after initial surgery (mitotane group) and 52 were not treated with any adjuvant treatment following surgery (control group). The median RFS was 36.5 months (4-199) in the mitotane 
group and 21 months $(4-180)$ in the control group $(p<0.001)$. The difference in OS was not significantly different between treated and untreated patients; however, in the group of patients with either stage III ACC or ki67 index $>10 \%$ adjuvant mitotane was associated with a significant increase of RFS $(p=0.014)$ (53). Interestingly, hormonesecreting ACC showed a higher ki67 index and this may explain why hormone secretion was a worse prognostic factor, as previously observed (54). In our experience, a low-dose mitotane regimen had acceptable toxicity and most patients were able to tolerate a long-term treatment. Therefore, this study is the first to support, although on a retrospective basis, the current recommendation of adjuvant mitotane therapy in ACC patients at high risk of recurrence following radical surgery (53).

Controversy on adjuvant mitotane is deemed to continue unless results of prospective controlled studies become available. Therefore, we have launched the first randomized trial in an adjuvant setting for ACC, the ADIUVO study (http://www.adiuvotrial.org) under the endorsement of the European Network for the Study of Adrenal Tumors (ENSAT). The aim of the study is to assess the efficacy of adjuvant mitotane treatment in prolonging RFS in ACC patients at low-intermediate risk of recurrence. Results of ADIUVO are expected in 2020.

A second prospective, randomized study on adjuvant therapy is currently under way. The ADIUVO-2 study aims to compare the efficacy of mitotane versus mitotane plus cisplatin and etoposide in preventing ACC recurrence following surgery in high-risk patients (ClinicalTrials.gov Identifier: NCT03583710).

\section{Practice Point}

- Mitotane reduces the risk of ACC recurrence following surgery; however, we are struggling to understand which patients are the best candidates for treatment.

\section{Research Agenda}

- Understand which patients truly respond to adjuvant mitotane therapy.

- $\quad$ Develop alternative adjuvant therapies. 


\section{Practical advices on management of adjuvant mitotane}

At the San Luigi Hospital, we start adjunctive mitotane treatment as soon as possible after surgery, at the very last within 3 months, in patients at high risk of recurrence, while low-risk patients have been enrolled in the ADIUVO trial (Figure 1).

We do not institute mitotane therapy before surgery and we rarely combine mitotane with cisplatin therapy when we perceive the risk of recurrence as very high. We associate adjuvant mitotane with local radiotherapy, limiting the combined therapy in patients with R1 surgery. The problem with this approach, which has a sound rationale, is the increased toxicity of the combination that only fit patients can sustain. In our practice, we use a low-dose mitotane regimen, with a starting dose of $1 \mathrm{~g}$ daily with daily increments of $0.5 \mathrm{~g}$ ( 1 tablet) every 4 days until the maximal tolerated dose, usually less $\leq 6 \mathrm{~g} /$ daily (Table 1 ).

We believe that a low-dose regimen is better tolerated with less impact on the quality of life of the patients, and this may eventually result in better compliance and more effective treatment. Although some experts advocate giving mitotane according to a high, rapidly escalating dose scheme (up to 6-9 g daily) (55), a recent survey of current practice in Italy showed that almost all expert centers use a low-dose regimen (56). By using this approach, mitotane was well tolerated and less than $5 \%$ of patients treated for at least 6 months had to discontinue permanently treatment for toxicity (early discontinuation was not recorded in the study). The trade-off with a very cautious drug escalation was the long time needed to attain therapeutic plasma concentrations of mitotane, averaging 8 months. Mitotane concentrations were significantly associated with the given dose and BMI, and women were treated with less doses (56). In analogy with the concept of time in target range used to evaluate the optimal exposure to warfarin therapy, we applied this measure to serial samplings of plasma mitotane in our series, and found that the time in target range was significantly associated with prolonged RFS. This result strengthens the value of mitotane monitoring and the concept of therapeutic mitotane concentrations in the adjuvant setting (56).

The most common unwanted effects are gastrointestinal manifestations that appear early, independently on mitotane levels. They can be managed with temporary dose reduction, or delay of dose increments, and supportive therapy. Elevated $\gamma$ glutamyltransferase levels are also frequently observed but are not actually troublesome unless values are exceedingly elevated. We usually do not reduce 
mitotane doses because GGT is elevated, given that this is the only altered liver function test. Clinically significant liver toxicity is characterized by a marked increase in transaminases and bilirubin, but is infrequently observed in the absence of predisposing conditions (43). We have seen rarely severe mitotane-induced hepatitis after few weeks of mitotane therapy, a time course that may suggest an idiosyncratic pathogenesis. Central neurologic toxicity (cerebellar symptoms, disturbed cognitive performance) is more closely associated with elevated mitotane concentrations (20 $\mathrm{mg} / \mathrm{L}$ ) but subtler symptoms, such as memory impairment or attention deficit, may be observed in some patients even at lower drug concentrations (35, 37, 43). A great individual variability in the susceptibility to mitotane-related unwanted effects is apparent for causes that are still unknown.

A general measure to deal with mitotane toxicity is a step down to the previously tolerated dose, or temporary drug withdrawal in the event of severe manifestations (Table 2). However, well-informed and motivated patients are able to cope with side effects and maintain compliance to treatment $(2,3,57)$. To accomplish this task, it is important to establish a close patient-physician relationship to induce and maintain adherence to treatment. Patients seek advice frequently, also because their local physicians are unfamiliar with mitotane use and its attendant complications, and it is necessary to give a timely counseling to keep patients on treatment. It is impossible to get adequate compliance to treatment when acting solely as consultants, who see patients every some months. It is necessary to stay in contact with the patients and communicate with them in a timely manner to help them dealing with the drug-related unwanted effects.

Because of the adrenolytic effect of mitotane, all patients should receive glucocorticoid replacement to prevent adrenal insufficiency (Table 2). Steroid doses are typically higher than in Addison's disease, due to an enhanced metabolic clearance rate of glucocorticoids induced by mitotane $(2,3,22,57,58)$. An inadequate treatment of adrenal insufficiency increases mitotane-related toxicity, particularly gastrointestinal side effects, and reduces tolerance $(2,3,43)$. Mineralocorticoid supplementation is not mandatory in all patients because the zona glomerulosa is partly spared by the toxic effect of mitotane (43). Hormone measurement is of little help in guiding steroid replacement, although high renin values, particularly if in combination with elevated potassium and postural hypotension and fatigue, may herald the need of mineralocorticoid supplementation. Measurements of urinary free cortisol, or serum 
cortisol, are of limited value and also salivary cortisol does not add meaningful information (59). We use exceedingly high ACTH levels as a potential marker of insufficient glucocorticoid coverage, since mitotane also inhibits pituitary ACTH secretion, and ACTH levels in mitotane-treated patients are usually far less elevated than in classic Addison's disease (59).

Moreover, mitotane affects thyroid and gonadal function in a complex way by mechanisms that imply inhibition of pituitary TSH secretion (60). Mitotane administration is associated with low FT4 levels without a compensatory rise in TSH, an effect that becomes apparent early in the course of treatment in almost half of patients. We are currently suggesting thyroxin replacement in these patients, and sometimes further thyroxin dose increments are needed to keep levels into the normal range. We hope that thyroxin replacement may serve to reduce some mental effects (i.e. concentration deficits) that patients on mitotane complain of. However, we do not still have prove of a clear-cut benefit of this measure.

In women, gonadal function is usually preserved and most female patients have regular cycles. We had indeed some conceptions, and even uneventful deliveries, during mitotane, despite that we strongly discourage to become pregnant. However, we do not recommend contraceptive pills, because they may be less effective and for the fear that estrogens may facilitate tumor progression (49). However, some women report disturbing spotting and heavy menstrual bleeding that are difficult to manage. Detection of ovarian cysts is frequent in such women and sometimes these lesions grow significantly and should be surgically treated (61).

In the male sex, mitotane treatment may cause sexual dysfunction as a late unwanted effect, in about one third of patients, due to inhibition of testosterone secretion. Mitotane-induced increase in SHBG may confound interpretation of hormonal data (57). Sex steroid replacement may become necessary to treat hypogonadism but worsens gynecomastia $(2,3,43,57)$.

Mitotane treatment is frequently associated with an alteration of the lipid panel, including elevation of both LDL and HDL cholesterol, which may reach remarkably high levels in some patients (57). The value of statin treatment is presently unclear (49).

The availability of mitotane measurement across Europe, as a free service offered by the company distributing mitotane (info@lysodren-europe.com), gives the possibility to guide dose adjustments and prevent severe toxicity. Mitotane monitoring is key for an 
appropriate management of adjuvant treatment to reach mitotane concentrations that have been associated with therapeutic effect.

The optimal duration of therapy remains undefined. The time to first recurrence after complete tumor resection is highly variable from some months to more than 10 years, but most recurrences occur within 2 years of primary surgery $(2,3,28,38,43)$. In our own series, about $70 \%$ of relapses took place in the first 2 years of follow-up, whereas the frequency of late (>5 years) relapses was less than $1 \%(44)$. It is our current practice to accommodate patient preferences between a range of possibilities (2 to 5 years of therapy) in a shared decision-making depending on tumor and patient characteristics. However, we are eager to prolong treatment, if well tolerated, in patients at elevated risk and we maintain treatment indefinitely after removal of a recurrence, or after radical surgery of stage IV ACC.

\section{Practice Points}

- Employ a low-dose, monitored mitotane regimen to reduce the risk of systemic toxicity and increase patient compliance.

- Do a careful follow-up of patients on adjuvant mitotane and treat proactively the unwanted effects of treatment.

\section{Research Agenda}

- Understand what is the optimal duration of adjuvant mitotane therapy.

- Understand what is the best supportive therapy to deal with drug toxicity. 


\section{Selection of patients to adjuvant mitotane}

Despite the limits of the available evidence, adjuvant mitotane therapy is currently recommended in many expert centers whenever the patients present an elevated risk of recurrence (Figure 1).

Differences do exist in the criteria used to define a high-risk condition. There is general agreement on stage I-II, complete (R0) resection and ki-67<10\% as markers of good prognosis, but opinions diverge whether stage III RO ACC should be associated with high risk (49). By the ENSAT ACC staging system, stage III applies to locally invasive tumors characterized by infiltration in surrounding tissue, positive regional lymph nodes or a neoplastic thrombus in the vena cava or vena renalis (5). It is biologically plausible that tumor spread in regional lymph nodes or in the vein system may portend to higher risk of recurrence than local infiltration and it is our opinion that subgroups at different risk of recurrence do exist among stage III ACC. Infrequently, a stage IV ACC, defined by presence of distant metastases (5), may be completely resected and has to be considered at high risk of recurrence.

The recent ESE-ENSAT guidelines have adopted the view that a low-risk condition is defined by stage I-II ACC, RO and ki67 <10\% (49). According to the guidelines, adjuvant mitotane is a non-mandatory option in patients at good prognosis, whereas a weak recommendation for adjuvant mitotane was done in the high-risk category (62).

Recent data suggest that the proliferation activity of the tumor is the most important factor predicting risk of recurrence following RO surgery. Assessment of the proliferation index ki-67 is currently used to assess proliferation, despite some problems to harmonize immunohistochemical readings among different pathologists. In a European multicentric study, a threshold value at $10 \%$ was found to separate patients at good or worse prognosis with a hazard ratio of recurrence of 1.042 per each \% increase (31). Given the availability of a large patient cohort totaling more than 500 patients, these are solid data confirming the view that tumor proliferation is a strong determinant of patient survival. The value of ACC proliferation has been already appreciated in smaller series by the use of mitosis count $(28,30)$, which is likely the single most predictive factor of Weiss score. Conversely, Weiss score per se does not clearly indicate the probability of tumor recurrence $(63,64)$.

Resection status is another established adverse risk factor, being Rx (unknown), R1 (microscopically positive margins) and R2 (macroscopically positive margins) associated with progressively reduced RFS irrespectively of other risk factors $(2,3,43$, 
64-66). However, it may be challenging to assess precisely resection status of a very large tumor, and pathologists infrequently report this parameter.

A number of molecular markers might potentially emerge in the future as powerful outcome predictors (67), but none of them has yet found a place in current practice. The major problem is that they can be evaluated only in research labs with complex techniques that are not readily available. Moreover, molecular analyses are timeconsuming and still not apt to give results in a time frame suitable for the decision making process. A recent study showed that hypermethylation and silencing of G0S2 is a hallmark of poor prognosis (68). Interestingly, assessment of G0S2 methylation can be done with a relatively simple and fast method that is potentially applicable in clinics. The combined assessment of G0S2 methylation with validated biomarkers (BUB1B-PINK1) may allow stratification of patients into different risk categories of ACC recurrence following surgery to guide allocation of patients to adjuvant therapy (68).

\section{Practice Points}

- Use adjuvant mitotane therapy when the risk of ACC recurrence is perceived to be high.

\section{Research Agenda}

- Develop molecular markers that allow improved prognostication to do a better selection of patients in need of treatment.

- Develop molecular markers that predict response to treatment. 


\section{Mitotane for advanced adrenocortical carcinoma}

The management of ACC patients with metastatic disease is challenging and the prognosis is generally poor, since most patients are doomed to die of disease progression within 1-2 years. However, ACC is a heterogeneous disease and a subset of patients bearing less aggressive tumors may experience quite prolonged survival. Several prognostic factors such as time since initial surgery, presence of distant metastases, number of metastatic lesions and number of organs involved, and high tumor grade have been found to predict survival in patients with metastatic ACC $(2,3$, 49, 69). Moreover, studies indicated that overt cortisol excess is associated with a detrimental prognosis due to the associated morbidity (54).

Treatment of advanced/metastatic patients may include as therapeutic tools locoregional approaches, such as surgery, radiofrequency ablation and chemoembolization, in patients with slowly progressive disease and low metastatic burden $(25,70,71)$. Isolated loco-regional recurrence, or oligo-metastatic disease, may be managed by surgery whenever complete resection (R0) can be envisaged. In this context, an aggressive surgical approach may lead to improved survival (48). Conversely, tumor debulking offers little benefit and is conceivable only in patients with functional tumors not controlled by medical treatment. Loco-regional measures may synergize with systemic therapies to attain long-lasting tumor control in patients with less aggressive ACC (49).

In presence of low-grade ACC with limited metastatic burden single-agent mitotane is a reasonable option (49) (Figure 2). Early studies assessing the efficacy of singleagent mitotane reported a rate of objective tumor responses between $13 \%$ and $31 \%$ $(37,39,72)$. However, most of the responses were of limited duration and complete responses were rarely observed. The outcome of these studies raised the concept of a "therapeutic range" of plasma mitotane concentrations that should be targeted in any patient with advanced/metastatic ACC. As a matter of fact, disease responses were mainly confined in patients attaining plasma mitotane concentration between 14 and $20 \mathrm{mg} / \mathrm{L}(37,39,72)$.

This concept has been validated more recently in a retrospective series of 127 patients receiving mitotane monotherapy for advanced ACC (73). In this study, the patients who reached a peak mitotane concentration $>14 \mathrm{mg} / \mathrm{L}$ had longer progression-free survival (PFS) and OS than patients who did not. The rate of objective responses was also higher in patients with mitotane concentrations at target, even if some responses 
were observed with lower levels. Overall, a complete response was observed in $2.3 \%$ of patients, a partial response in $18.1 \%$, and disease stabilization in $25.2 \%$, respectively. Interestingly, objective tumor responses were as high as $30 \%$ in patients with either low tumor burden ( $<10$ tumor lesions) or longer RFS after primary surgery ( $\geq 360$ days). Although the OS was quite limited, being 18.5 months, mitotane monotherapy was able to attain long-lasting tumor control in a number of patients (73). Therefore, the study supports the concept that mitotane monotherapy is indicated in the management of patients with a low tumor burden and/or more indolent disease. Some experts actually suggest achieving even higher mitotane concentrations, if tolerated.

Very recently, the outcome of single-agent monotherapy has been reported in 36 patients with metastatic ACC treated at a single center. In this retrospective series, most patients progressed under treatment but a remarkable $8 \%$ complete response rate was observed. The responding patients had non-functioning tumors with a lowvolume disease (74). Although on a limited patient cohort, the study confirms that mitotane monotherapy may be effective in selected ACC patients.

Besides its antitumor effect, mitotane is a strong inhibitor of adrenal steroidogenesis and it has a compelling indication in patients with endocrine symptoms, although the rate of success in controlling hormone excess is not well known $(2,3,66)$.

A classic management strategy implies mitotane monotherapy in the management of patients with a low tumor burden and/or more indolent disease, since mitotane is a slow acting drug (Figure 2). The latency of action of mitotane is related to the time needed to attain plasma concentrations at target. This particular feature of the drug translates in uncertainties concerning how long clinicians should wait to assess the efficacy of mitotane therapy, or its lack of. As a consequence, mitotane is continued almost indefinitely in many patients with advanced ACC lacking clear rules for its discontinuation.

A recent study aimed to clarify this issue, evaluating the time until a partial response was attained in patients with metastatic ACC (75). A cohort of 68 patients who survived more than 24 months after diagnosis of stage IV ACC was retrospectively analyzed, including 57 patients treated with mitotane monotherapy and the remainders with mitotane associated to chemotherapy. In this selected cohort of long-term survivors, almost all objective responses were observed within 12 months from start of mitotane and this finding suggests that mitotane may be discontinued after that time if 
ACC continues to progress. The value of the therapeutic range was reaffirmed, since responses were more frequently and rapidly observed in patients attaining mitotane concentrations $>14 \mathrm{mg} / \mathrm{L}$. The association between response and target mitotane concentrations was less apparent when mitotane was used with chemotherapy (75).

Chemotherapy plus mitotane is currently recommended for patients with highly proliferating and widespread disease (Figure 2). Chemotherapy in the management of advanced ACC is usually administered in association with mitotane not only in patients with de novo stage IV ACC, but also in patients with disease progression to mitotane therapy, when mitotane is usually maintained, if tolerated (49). Despite that combining mitotane with classic cytotoxic agents is a commonly used strategy, the evidence supporting a synergism between mitotane and chemotherapy is weak. However, indirect comparisons of response rates obtained in non-randomized phase II trials showed greater activity of chemotherapy regimens including mitotane (49). Up to now, no randomized study has tested prospectively the efficacy of mitotane plus chemotherapy versus mitotane alone.

The first prospective multinational trial on treatment of ACC (FIRM-ACT) ever published has recently set a standard of care for advanced/metastatic ACC (13). In this trial, the association of etoposide, doxorubicin and cisplatin plus mitotane (EDP-M) was found to be superior to streptozotocin plus mitotane in terms of disease response rate and PFS. On the bases of the results of this study, the EDP-M scheme is actually recommended as the standard approach for ACC patients by ESE-ENSAT guidelines (49). The efficacy of EDP-M in this multinational Phase III trial, however, was modest: the response rate was low (23\%), and the median PFS and OS were of only 5 and 14.8 months, respectively. The FIRM-ACT trial also provided some evidence that mitotane levels at target could improve patient outcome (13).

Mitotane efficacy, as previously discussed, is not immediate, and the so-called therapeutic range is usually attained within 2-3 months, so disease progression may precede the time when mitotane levels are at target. Chemotherapy may be effective in the first weeks of therapy and this is a pragmatic point favoring a functional synergism between mitotane and chemotherapy in patients with aggressive disease. On the other hand, mitotane may be also important for the long-term disease control. In the randomized trial FIRM-ACT, a few patients were free of progression after 4 years in both EDP-M and SZ-M arms. In these patients, mitotane could have contributed to the long-term delay of disease progression. In our experience, a few 
patients achieve disease response with the EDP-M regimen and then a long-lasting disease control by sustained mitotane therapy. The best outcome is observed in patients whose disease become surgically treatable following tumor shrinkage induced by chemotherapy and are able to attain a RO resection, and then undergo chronic mitotane treatment. However, the toxicity associated with the EDP-M scheme is important and only fit patients can sustain treatment. In case of compromised conditions and/or elder patients, we opt for mitotane monotherapy, or to mitotane plus cisplatin as an alternative to the whole EDP scheme.

\section{Practice Points}

- Use mitotane monotherapy in patients with limited tumor burden, and/or slow progression rate.

- Use mitotane plus chemotherapy (EDP) in patients with widespread disease and/or rapid progression rate, or when ACC progress to mitotane monotherapy.

- Consider associating loco-regional measures to systemic mitotane therapy to improve disease control.

\section{Research Agenda}

- Develop better treatment of advanced ACC.

- Develop molecular markers that predict response to treatment. 


\section{Conclusion}

Whenever ACC is completely removed, we should face the dilemma to use adjuvant therapy or not. In our opinion, adjuvant mitotane is the preferable approach in most cases, because the majority of patients referred to our institution following surgery for ACC show an elevated risk of recurrent disease. A better understanding of factors that influence prognosis and response to treatment (68) will help in stratifying patients according to their probability of benefiting from adjuvant mitotane, with the aim of sparing unnecessary toxicity to patients who are less likely to respond.

The strategy of treatment of advanced ACC is chosen considering a number of prognostic factors (tumor burden, type of progression, secretion, proliferation index) and the patient conditions. If a patient is fit and carries poor prognostic factors, we recommend the EDP-M regimen. Patients at perceived good prognosis may be treated with mitotane monotherapy and EDP is added on in case of disease progression. However, until significant advancements will take place, we have to deal with uncertainty using our best clinical judgment and personal experience in the clinical decision process for both adjuvant and palliative settings. 


\section{REFERENCES}

1. Kerkhofs TM, Verhoeven RH, Van der Zwan JM, et al. Adrenocortical carcinoma: a population-based study on incidence and survival in the Netherlands since 1993. Eur J Cancer. 2013; 49:2579-86.

2. Fassnacht $M$, Kroiss $M$, and Allolio B. Update in adrenocortical carcinoma. J Clin Endocrinol Metab. 2013; 98:4551-64.

3. Fassnacht $M$, Libé $R$, Kroiss $M$, and Allolio B. Adrenocortical carcinoma: a clinician's update. Nat Rev Endocrinol. 2011; 7:323-35.

4. Stigliano A, Chiodini I, Giordano R, et al. Management of adrenocortical carcinoma: a consensus statement of the Italian Society of Endocrinology (SIE). $J$ Endocrinol Invest. 2016; 39:103-21.

5. Fassnacht M, Johanssen S, Quinkler M, et al. Limited prognostic value of the 2004 International Union Against Cancer staging classification for adrenocortical carcinoma: proposal for a Revised TNM Classification. Cancer. 2009; 115:243-50.

6. Terzolo M, Daffara F, Ardito A, et al. Management of adrenal cancer: a 2013 update. J Endocrinol Invest. 2014; 37:207-17.

7. Puglisi S, Perotti P, Pia A, et al. Adrenocortical Carcinoma with Hypercortisolism. Endocrinol Metab Clin North Am. 2018; 47:395-407.

8. Terzolo M, Stigliano A, Chiodini I, et al. AME position statement on adrenal incidentaloma. Eur J Endocrinol. 2011; 164:851-70.

9. Crucitti F, Bellantone R, Ferrante A, et al. The Italian Registry for Adrenal Cortical Carcinoma: analysis of a multiinstitutional series of 129 patients. The ACC Italian Registry Study Group. Surgery. 1996; 119:161-70.

10. Gaujoux S, Mihai R, and joint working group of ESE and ENSAT. European Society of Endocrine Surgeons (ESES) and European Network for the Study of Adrenal Tumours (ENSAT) recommendations for the surgical management of adrenocortical carcinoma. Br J Surg. 2017; 104:358-76.

11. Amini N, Margonis GA, Kim Y, et al. Curative Resection of Adrenocortical Carcinoma: Rates and Patterns of Postoperative Recurrence. Ann Surg Oncol. 2016; 23:126-33.

12. Lombardi CP, Raffaelli M, Boniardi M, et al. Adrenocortical carcinoma: effect of hospital volume on patient outcome. Langenbecks Arch Surg. 2012; 397:201-7. 
13. Fassnacht $\mathrm{M}$, Terzolo $\mathrm{M}$, Allolio $\mathrm{B}$, et al. Combination chemotherapy in advanced adrenocortical carcinoma. N Engl J Med. 2012; 366:2189-97.

\section{${ }^{* *}$ The first randomized controlled trial on treatment of advanced ACC that set EDP-M as standard of therapy.}

14. Nelson AA, and Woodard G. Severe adrenal cortical atrophy (cytotoxic) and hepatic damage produced in dogs by feeding 2,2-bis(parachlorophenyl)-1,1-dichloroethane (DDD or TDE). Arch Pathol (Chic). 1949; 48:387-94.

15. Hart MM, Reagan RL, and Adamson $\mathrm{RH}$. The effect of isomers of DDD on the ACTH-induced steroid output, histology and ultrastructure of the dog adrenal cortex. Toxicol Appl Pharmacol. 1973; 24:101-13.

16. Schteingart DE. Conventional and novel strategies in the treatment of adrenocortical cancer. Braz J Med Biol Res. 2000; 33:1197-200.

17. Trainer PJ, and Besser M. Cushing's syndrome. Therapy directed at the adrenal glands. Endocrinol Metab Clin North Am. 1994; 23:571-84.

18. Lin $\mathrm{CW}$, Chang $\mathrm{YH}$, and $\mathrm{Pu} \mathrm{HF}$. Mitotane exhibits dual effects on steroidogenic enzymes gene transcription under basal and cAMP-stimulating microenvironments in NCl-H295 cells. Toxicology. 2012; 298:14-23.

19. Zsippai A, Szabó DR, Tömböl Z, et al. Effects of mitotane on gene expression in the adrenocortical cell line NCl-H295R: a microarray study. Pharmacogenomics. 2012; 13:1351-61.

20. Lehmann TP, Wrzesiński T, and Jagodziński PP. The effect of mitotane on viability, steroidogenesis and gene expression in NCl-H295R adrenocortical cells. Mol Med Rep. 2013; 7:893-900.

21. Hescot $S$, Slama $A$, Lombès $A$, et al. Mitotane alters mitochondrial respiratory chain activity by inducing cytochrome c oxidase defect in human adrenocortical cells. Endocr Relat Cancer. 2013; 20:371-81.

22. Chortis V, Taylor AE, Schneider P, et al. Mitotane therapy in adrenocortical cancer induces CYP3A4 and inhibits 5a-reductase, explaining the need for personalized glucocorticoid and androgen replacement. J Clin Endocrinol Metab. 2013; 98:16171.

23. Sbiera S, Leich E, Liebisch G, et al. Mitotane Inhibits Sterol-O-Acyl Transferase 1 Triggering Lipid-Mediated Endoplasmic Reticulum Stress and Apoptosis in Adrenocortical Carcinoma Cells. Endocrinology. 2015; 156:3895-908. 
24. Hermsen IG, Kerkhofs TM, den Butter G, et al. Surgery in adrenocortical carcinoma: Importance of national cooperation and centralized surgery. Surgery. 2012; 152:50-6.

25. Bellantone R, Ferrante A, Boscherini M, et al. Role of reoperation in recurrence of adrenal cortical carcinoma: results from 188 cases collected in the Italian National Registry for Adrenal Cortical Carcinoma. Surgery. 1997; 122:1212-8.

26. Icard P, Goudet P, Charpenay $\mathrm{C}$, et al. Adrenocortical carcinomas: surgical trends and results of a 253-patient series from the French Association of Endocrine Surgeons study group. World J Surg. 2001; 25:891-7.

27. Schulick RD, and Brennan MF. Long-term survival after complete resection and repeat resection in patients with adrenocortical carcinoma. Ann Surg Oncol. 1999; 6:719-26.

28. Stojadinovic A, Ghossein RA, Hoos A, et al. Adrenocortical carcinoma: clinical, morphologic, and molecular characterization. J Clin Oncol. 2002; 20:941-50.

29. Abiven G, Coste J, Groussin L, et al. Clinical and biological features in the prognosis of adrenocortical cancer: poor outcome of cortisol-secreting tumors in a series of 202 consecutive patients. J Clin Endocrinol Metab. 2006; 91:2650-5.

30. Volante $M$, Bollito $E$, Sperone $P$, et al. Clinicopathological study of a series of 92 adrenocortical carcinomas: from a proposal of simplified diagnostic algorithm to prognostic stratification. Histopathology. 2009; 55:535-43.

31. Beuschlein F, Weigel J, Saeger W, et al. Major prognostic role of Ki67 in localized adrenocortical carcinoma after complete resection. J Clin Endocrinol Metab. 2015; 100:841-9.

\section{${ }^{* *}$ The largest study on ACC prognostication establishing the role of ki67 proliferation index as the best marker of the risk of recurrence following surgery.}

32. Schteingart DE, Motazedi A, Noonan RA, and Thompson NW. Treatment of adrenal carcinomas. Arch Surg. 1982; 117:1142-6.

33. Bodie B, Novick AC, Pontes JE, et al. The Cleveland Clinic experience with adrenal cortical carcinoma. J Urol. 1989; 141:257-60.

34. Kasperlik-Załuska AA, Migdalska BM, Zgliczyński S, and Makowska AM. Adrenocortical carcinoma. A clinical study and treatment results of 52 patients. Cancer. 1995; 75:2587-91. 
35. Kasperlik-Zaluska AA. Clinical results of the use of mitotane for adrenocortical carcinoma. Braz J Med Biol Res. 2000; 33:1191-6.

36. Dickstein G, Shechner C, Arad E, et al. Is there a role for low doses of mitotane (o,p'-DDD) as adjuvant therapy in adrenocortical carcinoma? J Clin Endocrinol Metab. 1998; 83:3100-3.

37. Baudin E, Pellegriti G, Bonnay M, et al. Impact of monitoring plasma 1,1dichlorodiphenildichloroethane (o,p'DDD) levels on the treatment of patients with adrenocortical carcinoma. Cancer. 2001; 92:1385-92.

38. Pommier RF, and Brennan MF. An eleven-year experience with adrenocortical carcinoma. Surgery. 1992; 112:963-70; discussion 70-1.

39. Haak HR, Hermans J, van de Velde CJ, et al. Optimal treatment of adrenocortical carcinoma with mitotane: results in a consecutive series of 96 patients. $\mathrm{Br} \mathrm{J}$ Cancer. 1994; 69:947-51.

40. Vassilopoulou-Sellin R, Guinee VF, Klein MJ, et al. Impact of adjuvant mitotane on the clinical course of patients with adrenocortical cancer. Cancer. 1993; 71:311923.

41. Barzon L, Fallo F, Sonino N, et al. Adrenocortical carcinoma: experience in 45 patients. Oncology. 1997; 54:490-6.

42. Schteingart DE, Doherty GM, Gauger PG, et al. Management of patients with adrenal cancer: recommendations of an international consensus conference. Endocr Relat Cancer. 2005; 12:667-80.

43. Terzolo M, and Berruti A. Adjunctive treatment of adrenocortical carcinoma. Curr Opin Endocrinol Diabetes Obes. 2008; 15:221-6.

44. Terzolo $M$, Angeli $A$, Fassnacht $M$, et al. Adjuvant mitotane treatment for adrenocortical carcinoma. N Engl J Med. 2007; 356:2372-80.

45. Huang $\mathrm{H}$, and Fojo $\mathrm{T}$. Adjuvant mitotane for adrenocortical cancer--a recurring controversy. J Clin Endocrinol Metab. 2008; 93:3730-2.

46. Grubbs EG, Callender GG, Xing Y, et al. Recurrence of adrenal cortical carcinoma following resection: surgery alone can achieve results equal to surgery plus mitotane. Ann Surg Oncol. 2010; 17:263-70.

47. Fassnacht M, Johanssen S, Fenske W, et al. Improved survival in patients with stage II adrenocortical carcinoma followed up prospectively by specialized centers. J Clin Endocrinol Metab. 2010; 95:4925-32. 
48. Wängberg B, Khorram-Manesh A, Jansson S, et al. The long-term survival in adrenocortical carcinoma with active surgical management and use of monitored mitotane. Endocr Relat Cancer. 2010; 17:265-72.

49. Fassnacht M, Dekkers OM, Else T, et al. European Society of Endocrinology Clinical Practice Guidelines on the management of adrenocortical carcinoma in adults, in collaboration with the European Network for the Study of Adrenal Tumors. Eur J Endocrinol. 2018; 179:G1-G46.

${ }^{* *}$ The most recent and comprehensive guidelines on ACC management to guide treatment in different clinical scenarios.

50. Berruti A, Grisanti S, Pulzer A, et al. Long-Term Outcomes of Adjuvant Mitotane Therapy in Patients With Radically Resected Adrenocortical Carcinoma. J Clin Endocrinol Metab. 2017; 102:1358-65.

**The best study on adjuvant mitotane treatment supporting a benefit of treatment in reducing the risk of recurrence following surgery.

51. Postlewait LM, Ethun CG, Tran TB, et al. Outcomes of Adjuvant Mitotane after Resection of Adrenocortical Carcinoma: A 13-Institution Study by the US Adrenocortical Carcinoma Group. J Am Coll Surg. 2016; 222:480-90.

52. Tang Y, Liu Z, Zou Z, et al. Benefits of Adjuvant Mitotane after Resection of Adrenocortical Carcinoma: A Systematic Review and Meta-Analysis. Biomed Res Int. 2018; 9362108.

*An interesting meta-analysis on post-operative adjuvant mitotane treatment supporting a benefit of treatment in prologing either RFS or OS.

53. Calabrese A, Basile V, Puglisi S, et al. Adjuvant mitotane therapy is beneficial in non-metastatic adrenocortical carcinoma at high risk of recurrence. Eur $J$ Endocrinol. 2019; 180:387-96.

*The most recent study on adjuvant mitotane treatment showing a benefit of treatment in reducing the risk of recurrence following surgery in high-risk ACC patients.

54. Berruti A, Fassnacht M, Haak $\mathrm{H}$, et al. Prognostic role of overt hypercortisolism in completely operated patients with adrenocortical cancer. Eur Urol. 2014; 65:832-8.

55. Faggiano A, Leboulleux S, Young J, et al. Rapidly progressing high o,p'DDD doses shorten the time required to reach the therapeutic threshold with an acceptable tolerance: preliminary results. Clin Endocrinol (Oxf). 2006; 64:110-3. 
56. Puglisi S, Calabrese A, Basile V, et al. Mitotane Concentrations Influence the Risk of Recurrence in Adrenocortical Carcinoma Patients on Adjuvant Treatment. $J$ Clin Med. 2019; 8.

*The most recent study on the value of monitoring mitotane concentrations in an adjuvant setting.

57. Daffara F, De Francia S, Reimondo G, et al. Prospective evaluation of mitotane toxicity in adrenocortical cancer patients treated adjuvantly. Endocr Relat Cancer. 2008; 15:1043-53.

58. Hague RV, May W, and Cullen DR. Hepatic microsomal enzyme induction and adrenal crisis due to o,p'DDD therapy for metastatic adrenocortical carcinoma. Clin Endocrinol (Oxf). 1989; 31:51-7.

59. Reimondo G, Puglisi S, Zaggia B, et al. Effects of mitotane on the hypothalamicpituitary-adrenal axis in patients with adrenocortical carcinoma. Eur $J$ Endocrinol. 2017; 177:361-7.

60. Zatelli MC, Gentilin E, Daffara F, et al. Therapeutic concentrations of mitotane (o,p'DDD) inhibit thyrotroph cell viability and TSH expression and secretion in a mouse cell line model. Endocrinology. 2010; 151:2453-61.

61. Salenave S, Bernard V, Do Cao C, et al. Ovarian macrocysts and gonadotropeovarian axis disruption in premenopausal women receiving mitotane for adrenocortical carcinoma or Cushing's disease. Eur J Endocrinol. 2015; 172:141-9.

62. Berruti A, Fassnacht $M$, Baudin E, et al. Adjuvant therapy in patients with adrenocortical carcinoma: a position of an international panel. J Clin Oncol. 2010; 28:e401-2.

63. Beuschlein F, Obracay J, Saeger W, et al. Prognostic value of histological markers in localized adrenocortical carcinoma after complete resection. Experimental and Clinical Endocrinology \& Diabetes. 2013; 121:OP8_49.

64. Papotti M, Libè R, Duregon $\mathrm{E}$, et al. The Weiss score and beyond--histopathology for adrenocortical carcinoma. Horm Cancer. 2011; 2:333-40.

65. Kebebew E, Reiff E, Duh QY, et al. Extent of disease at presentation and outcome for adrenocortical carcinoma: have we made progress? World J Surg. 2006; 30:872-8.

66. Bourdeau I, MacKenzie-Feder J, and Lacroix A. Recent advances in adrenocortical carcinoma in adults. Curr Opin Endocrinol Diabetes Obes. 2013; 20:192-7. 
67. Jouinot $A$, and Bertherat J. MANAGEMENT OF ENDOCRINE DISEASE: Adrenocortical carcinoma: differentiating the good from the poor prognosis tumors. Eur J Endocrinol. 2018; 178:R215-R30.

68. Mohan DR, Lerario AM, Else T, et al. Targeted Assessment of G0S2 methylation identifies a rapidly recurrent, routinely fatal molecular subtype of adrenocortical carcinoma. Clin Cancer Res. 2019; 25:3276-88.

\section{${ }^{* *} \mathrm{~A}$ recent addition of a promising biomarker to the list of molecular tools to predict the risk of recurrence following surgery.}

69. Malandrino P, Al Ghuzlan A, Castaing M, et al. Prognostic markers of survival after combined mitotane- and platinum-based chemotherapy in metastatic adrenocortical carcinoma. Endocr Relat Cancer. 2010; 17:797-807.

70. Wood BJ, Abraham J, Hvizda JL, et al. Radiofrequency ablation of adrenal tumors and adrenocortical carcinoma metastases. Cancer. 2003; 97:554-60.

71. Cazejust J, De Baère $\mathrm{T}$, Auperin A, et al. Transcatheter arterial chemoembolization for liver metastases in patients with adrenocortical carcinoma. J Vasc Interv Radiol. 2010; 21:1527-32.

72. Hermsen IG, Fassnacht M, Terzolo M, et al. Plasma concentrations of o,p'DDD, o,p'DDA, and o,p'DDE as predictors of tumor response to mitotane in adrenocortical carcinoma: results of a retrospective ENS@T multicenter study. J Clin Endocrinol Metab. 2011; 96:1844-51.

73. Megerle F, Herrmann W, Schloetelburg W, et al. Mitotane Monotherapy in Patients With Advanced Adrenocortical Carcinoma. J Clin Endocrinol Metab. 2018; 103:1686-95.

${ }^{* *}$ The best study on mitotane monotherapy in patients with advanced ACC supporting a benefit of treatment in patients with less aggressive tumors.

74. Reidy-Lagunes DL, Lung B, Untch BR, Raj N, Hrabovsky A, Kelly C, et al. Complete Responses to Mitotane in Metastatic Adrenocortical Carcinoma-A New Look at an Old Drug. Oncologist. 2017; 22:1102-6.

75. Vezzosi D, Do Cao C, Hescot S, et al. Time Until Partial Response in Metastatic Adrenocortical Carcinoma Long-Term Survivors. Horm Cancer. 2018; 9:62-9.

*The most recent study on mitotane treatment in patients with advanced ACC establishing criteria for assessing mitotane efficacy. 


\section{Legends}

Figure-1. Management of ACC patients following surgery with radical intent.

Figure-2. Management of ACC patients with advanced or recurrent disease not amenable of surgery with radical intent. 